\title{
Clinical and metabolic effects of first-line treatment with somatostatin analogues or surgery in acromegaly: a retrospective and comparative study
}

\author{
Carla Giordano - Alessandro Ciresi · Marco Calogero Amato • \\ Rosario Pivonello • Renata Simona Auriemma - Ludovica Francesca Stella Grasso • \\ Aldo Galluzzo • Annamaria Colao
}

Published online: 25 November 2011

(C) Springer Science+Business Media, LLC 2011

\begin{abstract}
To evaluate the metabolic effects of first-line somatostatin analogues or surgery in acromegaly. Retrospective, comparative, 12-month follow-up. Two hundred and thirty one patients (123 men, age $47.32 \pm 14.63$ years) with active acromegaly, first line treatments were somatostatin analogues in $151(65.4 \%)$ and surgery in 80 (34.6\%). Metabolic syndrome (MS) parameters, glucose, insulin and GH during oral glucose tolerance test, stimulated insulin sensitivity by insulin sensitivity index (ISI Matsuda), early and total insulin-secretion rate by insulinogenic index and $\mathrm{AUC}_{\mathrm{INS}}$, visceral adiposity function, expressed by visceral adipose index (VAI). Somatostatin analogues treatment improved all MS parameters and significantly reduced fasting glucose $(P<0.001)$, HbAlc $(P=0.014)$ and the prevalence of DM $(P=0.003)$ when disease control was achieved. Both somatostatin analogues and surgery improved ISI Matsuda $(P<0.001)$ and reduced $\mathrm{AUC}_{\mathrm{INS}}(P<0.001)$ and VAI $(P<0.001$ and $P=0.003$, respectively). Only in controlled somatostatin analogues-treated patients a significant reduction in insulinogenic index $(P=0.010)$ was observed. ISI Matsuda showed a significant independent correlation with IGF-1 levels $(\beta=-0.258 ; P=0.001)$ and VAI score $(\beta=-$ $0.430 ; P<0.001)$. VAI was independently correlated with IGF-1 $\quad(\beta=0.183 ; \quad P=0.004)$. Both somatostatin
\end{abstract}

C. Giordano · A. Ciresi - M. C. Amato · A. Galluzzo Department of Endocrinology and Metabolic Diseases, DOSAC, University of Palermo, piazza delle Cliniche 2, 90127 Palermo, Italy

R. Pivonello · R. S. Auriemma · L. F. S. Grasso · A. Colao ( $\square)$ Department of Molecular and Clinical Endocrinology and Oncology, Section of Endocrinology, University "Federico II" of Naples, via S. Pansini 5, 80131 Naples, Italy

e-mail: colao@unina.it analogues and surgery can safely be used as first-line therapy in acromegaly, without any untoward effects on glucose tolerance. The control of acromegaly is the main determinant of beneficial effects on general features of insulin sensitivity. VAI could represent an additional link between disease control and insulin sensitivity.

Keywords GH · IGF-I · Acromegaly $\cdot$ Pituitary · Glucose $\cdot$ Insulin

\section{Introduction}

Acromegaly is a rare disease characterized by excess secretion of growth hormone $(\mathrm{GH})$ and increased circulating insulin-like growth factor 1 (IGF-1) concentrations. In $90-95 \%$ of patients the disease is caused by a GH-secreting pituitary adenoma and the diagnosis is preceded by about 10 years of active unrecognized disease [1]. Chronic exposure to GH and IGF1 hypersecretion is associated with multisystem comorbidities, including cardiovascular disease, hypertension, sleep apnea syndrome, colon polyposis, arthropathy, and metabolic complications, leading to increased morbidity and premature mortality [2]. In patients with acromegaly alterations in glucose tolerance, such as impaired glucose tolerance (IGT), impaired fasting glucose (IFG) and overt type 2 diabetes mellitus $(\mathrm{DM})$, are more frequent than in the general population. The prevalence of DM or other carbohydrate metabolism disturbances in acromegaly ranges from 19 to $56 \%$ [3] and the presence of these comorbidities correlates with cardiovascular morbidity and mortality in acromegaly [4-6]. The therapeutic options for acromegaly include surgery, radiotherapy and medical therapies, such as dopamine agonists, somatotropin release-inhibiting factor receptor 
ligand (SRIF-RL) and the GH receptor antagonist pegvisomant [7]. Medical therapy is currently most widely used as secondary treatment for persistent or recurrent acromegaly following non-curative surgery, although it is increasingly used as primary therapy. Treatment with SRIF-RL induces control of GH and IGF-1 excess in the majority of patients [8-10]. SRIF-RL also exert different hormonal effects on pancreatic beta-cell insulin secretion and glucose homeostasis. Native somatostatin inhibits both insulin and glucagon secretion and decreases the gastrointestinal glucose absorption rate $[11,12]$. SRIF-RL treatment is believed to have similar effects, not only reducing insulin resistance (IR) but also impairing insulin secretion. In particular, Steffin et al. [13] suggested the use of secretagogue hypoglycemic agents rather than insulinsensitizers as choice treatment in diabetic acromegalic patients during SRIF-RL treatment. However, discordant data are reported on the effect of treatments regarding the glucose tolerance of acromegalic patients [14, 15]. In this setting, increased glucose levels in both controlled and uncontrolled patients were reported during SRIF-RL therapy and not after surgery in some studies [16-18] but not in others $[19,20]$. In a previous study of our group, we found deterioration of glucose tolerance only at beginning of SRIF-RL therapy [21]. In other studies, we found a similar deterioration in glucose tolerance after therapy with either SRIF-RL or surgical treatment, in relation to increased BMI, considered the major predictor in identifying those patients having impairment in glucose tolerance [22, 23]. Despite this rather large bulk of data, a more exhaustive analysis on metabolic effects determined by the different treatments in acromegaly is still needed.

The aim of the current study was to extend previous analysis on glucose tolerance in acromegaly by evaluating the effects of 12 months of first-line SRIF-RL or surgical treatment on each criterion of the metabolic syndrome (MS), insulin sensitivity and visceral adiposity index (VAI) [24], a new parameter indicating adipose tissue dysfunction associated with cardiometabolic risk, in a large group of acromegalic patients in relation to disease control.

\section{Patients and methods}

For the purpose of this study we reviewed all files from consecutive patients with active acromegaly coming to the Units of Endocrinology or Neurosurgery of the "Federico II" University of Naples and to the Unit of Endocrinology of the University of Palermo from January 1st 2000 to December 31st 2009, primarily treated with either surgery or depot SRIF-RL, i.e. lanreotide (LAN) or slow-release octreotide (LAR), and with an available follow-up of at least 12 months. The Naples group started a database including patients with pituitary tumors in 1997 in order to evaluate of the effects of the GH/IGF-I axis on the cardiovascular system approved by the local Ethical Committee (63/97). The Unit of Palermo included metabolic and hormonal data of patients from a database made to mainly evaluate the effects of the GH/IGF-I axis on the cardio-metabolic risk in relation to insulin resistance in acromegalic patients. Due to the study design, this is a non randomized study. However, our routine procedure generally considers first-line treatment with SRIF-RL for 6-12 months, unless the tumors are clearly non invasive on Magnetic Resonance Imaging (MRI) and/or the patients who do not present any surgical or anesthesiological risk [25].

\section{Inclusion criteria}

Patients treated with first-line surgery via trans-sphenoidal route by microscopic and/or endoscopic approach or with first-line depot SRIF-RL treatment, achieving control of the disease, and with available follow-up after 12 months of treatment. Patients with uncontrolled acromegaly remained into the study as internal control.

\section{Exclusion criteria}

Patients receiving combined dopamine-agonists and SRIFRL because of a mixed GH/PRL-secreting tumor, receiving the s.c. octreotide for longer than 15 days, requiring other medical treatment (pegvisomant, surgery or SRIF-RL as second-line adjuvant treatment before the completion of the 12 months), or with a follow-up shorter than 6 months after surgery or pharmacotherapy were excluded from this study.

\section{Patients}

Of 361 newly diagnosed patients affected by active acromegaly 231, 123 men $(53.2 \%)$ and 108 women (46.8\%), with mean age $47.32 \pm 14.63$ years (range: $20-82$ ), were included in this study (Fig. 1). Sitxy-nine patients (29.9\%) were already included into previous studies [17-20]. At the time of hospitalization, all patients signed an informed consent for the scientific use of their data. Diagnosis of active acromegaly was established on the basis of widely recognized criteria [26]. The duration of disease (95.6 \pm 69.7 months) was established by patient interview, patients' pictures, and onset of osteoarticular symptoms. Hypopituitarism, if present, was appropriately replaced before any testing was done. Table 1 shows the baseline clinical and biochemical features of the patients. 
Fig. 1 Flow-chart of patients distribution and inclusion into the study according with adopted criteria. $S R I F-R L$ somatotropin release-inhibiting factor receptor ligand (somatostatin analogues)

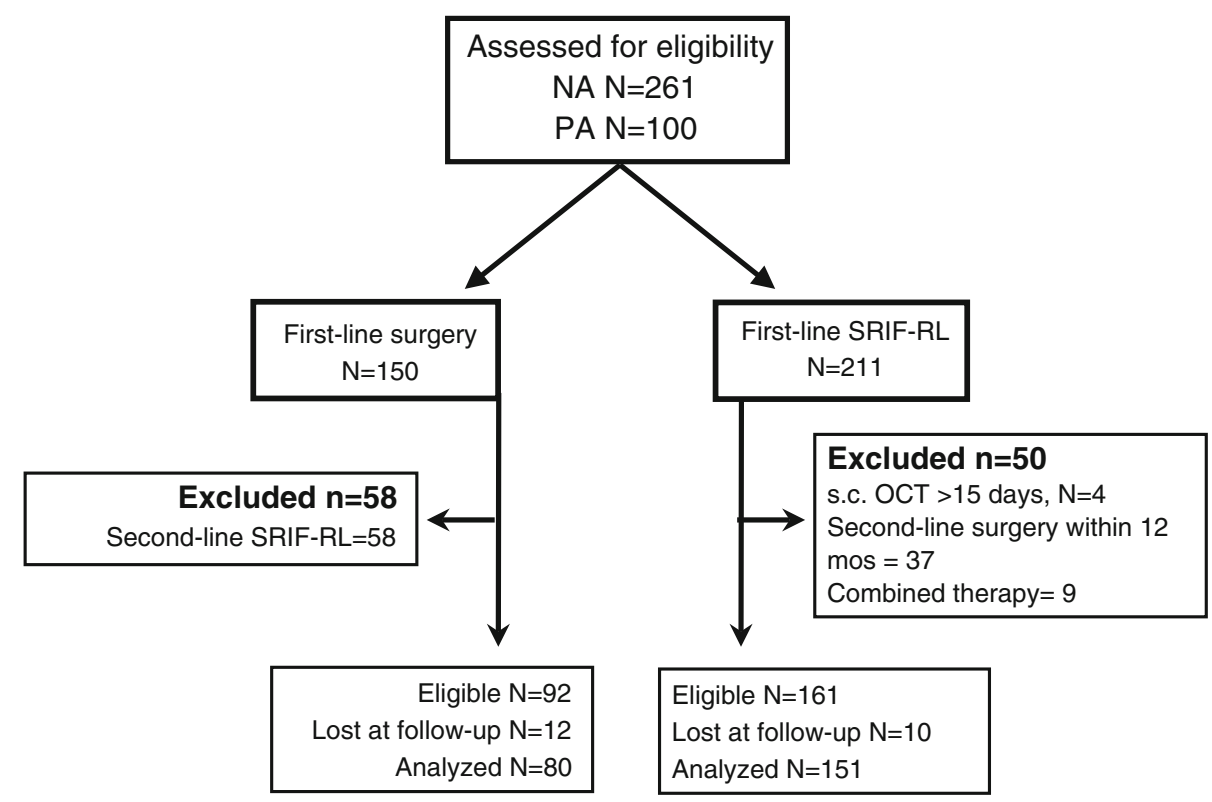

Study design

Males : VAI

$$
\begin{aligned}
= & {[\mathrm{WC} / 39.68+(1.88 \times \mathrm{BMI})] \times(\mathrm{TG} / 1.03) } \\
& \times(1.31 / \mathrm{HDL})
\end{aligned}
$$

at baseline and after 12 months of first-line medical or surgical treatment, underwent a complete clinical and metabolic evaluation. In all patients we measured systolic and diastolic blood pressure, waist circumference (WC), $\mathrm{BMI}$, lipid profile, $\mathrm{HbAlc}$, mean fasting plasma $\mathrm{GH}$ (at least three blood samples at 30-min intervals) and IGF-1 levels; then, to normalize IGF-1 for age in individual patients, we calculated the ratio between the IGF-1 level and the upper limit of the normal (ULN) range for age (normal $=\leq 1)$ and we showed the data as IGF-1 ULN. In line with Giustina et al. [27], patients were considered controlled in presence of IGF-I ULN $\leq 1$. An oral glucose tolerance test (OGTT) was performed after overnight fasting by measuring plasma blood glucose, insulin levels and GH every $30 \mathrm{~min}$ for $2 \mathrm{~h}$ after $75 \mathrm{~g}$ oral glucose load in all patients but 26 with overt diabetes. The areas under the curve of glucose $\left(\mathrm{AUC}_{\mathrm{GLU}}\right)$, insulin $\left(\mathrm{AUC}_{\mathrm{INS}}\right)$ and $\mathrm{GH}$ $\left(\mathrm{AUC}_{\mathrm{GH}}\right)$ during $2 \mathrm{~h}$ OGTT were also calculated. The diagnosis of diabetes or glucose tolerance abnormalities was made according to the American Association of Clinical Endocrinologists medical guidelines [28].

Basal insulin resistance (IR) was assessed using homeostasis model assessment of the insulin resistance (Homa-IR) index [29]. Stimulated insulin sensitivity was measured using the insulin sensitivity index (ISI), a composite index derived from the OGTT and validated by Matsuda et al. [30]. The early insulin-secretion rate was evaluated using the insulinogenic index [31], while total insulin secretion was assessed by $\mathrm{AUC}_{\mathrm{INS}}$. VAI score was calculated as described [24] using the following formula and was differentiated according to sex:

$$
\text { Females : } \begin{aligned}
& {[\mathrm{WC} / 36.58+(1.89 \times \mathrm{BMI})] \times(\mathrm{TG} / 0.81) } \\
\times & (1.52 / \mathrm{HDL})
\end{aligned}
$$

Of the 231 patients included into this study, 151 (65.4\%) were treated with first-line depot SRIF-RL for 12 months: $106(70.2 \%)$ received octreotide-long acting release (LAR) (10-40 mg every 28 days) and 45 (29.8\%) lanreotide-slow release (SR) (60 mg every 14 days) or autogel (ATG) (60-120 mg every 28 days). Octreotide was started at a dose of $20 \mathrm{mg} / 28$ days, lanreotide at a dose of $60 \mathrm{mg} /$ 14 days and dosages were titrated on the basis of GH and IGF-1, to obtain controlled levels, at week 12 or 24. In the octreotide-group 51 patients practiced the monthly dose of $20 \mathrm{mg}, 50$ patients $30 \mathrm{mg}$, 4 patients $10 \mathrm{mg}$ and 1 patient $40 \mathrm{mg}$; in the lanreotide-group, 11 patients had SR $60 \mathrm{mg} /$ 14 days, 20 the monthly dose of $120 \mathrm{mg}, 8$ patients $60 \mathrm{mg}$ and 6 patients $90 \mathrm{mg}$ of ATG.

The remaining 80 patients $(34.6 \%)$ underwent first-line pituitary surgery and were followed up for 12 months. Patients undergoing surgery but needing SRIF-RL as second-line adjuvant treatment before the completion of the 12 months were excluded. The 40 patients with unsuccessful surgery did not receive SRIF-RL for the following reasons: 8 patients refused to start medical treatment despite deserving a treatment post-surgery and spontaneously decided to wait since they felt they improved clinically, 21 patients had GH and IGF-I levels borderline so that we decided not to start any treatment before it was clear they had to be treated, 11 patients were not treated as they started with radiotherapy after surgery. As regards hypoglycaemic therapy, all IFG and IGT patients were 
Table 1 Baseline clinical and biochemical features of all patients grouped according to treatment assigned: somatostatin analogues (somatotropin release-inhibiting factor receptor ligand, SRIF-RL) (Group A) and surgery (Group B)

\begin{tabular}{|c|c|c|c|c|}
\hline & $\begin{array}{l}\text { All patients } \\
\text { (No. 231) }\end{array}$ & $\begin{array}{l}\text { SRIF-RL-treated } \\
\text { (Group A: No. 151) }\end{array}$ & $\begin{array}{l}\text { Surgery-treated } \\
\text { (Group B: No. 80) }\end{array}$ & $P$ \\
\hline \multicolumn{5}{|l|}{ Clinical characteristics } \\
\hline Age (year) & $47.32 \pm 14.63$ & $48.25 \pm 15.59$ & $45.58 \pm 12.53$ & 0.187 \\
\hline BMI $\left(\mathrm{kg} / \mathrm{m}^{2}\right)$ & $25.03 \pm 3.05$ & $25.35 \pm 3.16$ & $24.43 \pm 2.74$ & 0.028 \\
\hline \multicolumn{5}{|l|}{ Gender } \\
\hline Males & $123(53.2)$ & $84(55.6)$ & $39(48.8)$ & 0.319 \\
\hline Females & $108(46.8)$ & $67(44.4)$ & $41(51.3)$ & \\
\hline Family history for diabetes & $105(45.5)$ & $69(45.7)$ & $36(45)$ & 0.920 \\
\hline Duration of disease (months) & $95.55 \pm 69.73$ & $96.94 \pm 73.55$ & $92.93 \pm 62.25$ & 0.678 \\
\hline Tumor volume $\left(\mathrm{cm}^{3}\right)$ & $1.86 \pm 1.94$ & $1.66 \pm 1.49$ & $2.14 \pm 2.42$ & 0.089 \\
\hline Basal GH levels $(\mu \mathrm{g} / \mathrm{l})$ & $37.74 \pm 31.68$ & $37.89 \pm 32.64$ & $37.47 \pm 29.98$ & 0.925 \\
\hline Nadir GH levels $(\mu \mathrm{g} / \mathrm{l})$ & $24.97 \pm 23.30$ & $26.70 \pm 25.33$ & $22.21 \pm 19.44$ & 0.177 \\
\hline Basal IGF1 (ULN) & $2.39 \pm 0,77$ & $2.32 \pm 0.78$ & $2.52 \pm 0.74$ & 0.053 \\
\hline Metabolic syndrome & $85(36.8)$ & $59(39.1)$ & $26(32.5)$ & 0.324 \\
\hline Increased waist circumference & $53(22.9)$ & $29(19.2)$ & $24(30)$ & 0.063 \\
\hline Hypertriglyceridemia & $87(37.7)$ & $69(45.7)$ & $18(22.5)$ & 0.001 \\
\hline Low HDL cholesterol & $109(47.2)$ & $72(47.7)$ & $37(46.3)$ & 0.836 \\
\hline Increased systolic blood pressure or specific treatment & $164(71)$ & $106(70.2)$ & $58(72.5)$ & 0.714 \\
\hline Increased diastolic blood pressure or specific treatment & $115(49.8)$ & $73(48.3)$ & $42(52.5)$ & 0.548 \\
\hline \multicolumn{5}{|l|}{ Glucose tolerance } \\
\hline Normal tolerance & $145(62.7)$ & $98(64.9)$ & $47(58.8)$ & 0.358 \\
\hline Impaired fasting glucose (IFG) & $17(7.3)$ & $13(8.6)$ & $4(5)$ & 0.318 \\
\hline Impaired glucose tolerance (IGT) & $30(13)$ & $13(8.6)$ & $17(21.3)$ & 0.007 \\
\hline IFG + IGT & $8(3.5)$ & $4(2.6)$ & $4(5)$ & 0.453 \\
\hline Diabetes mellitus & $31(13.4)$ & $23(15.2)$ & $8(10)$ & 0.267 \\
\hline Homa IR & $4.24 \pm 2.59$ & $4.12 \pm 2.69$ & $4.46 \pm 2.41$ & 0.352 \\
\hline ISI Matsuda & $3.04 \pm 1.43$ & $3.27 \pm 1.67$ & $2.67 \pm 0.84$ & 0.004 \\
\hline $\mathrm{AUC}_{2 \mathrm{hInsulin}}$ & $8,738 \pm 3,450$ & $8,342 \pm 3,550$ & $9,359 \pm 3,212$ & 0.040 \\
\hline Insulinogenic index (Ins $30 \mathrm{~min} / \mathrm{Glu} 30 \mathrm{~min})$ & $35.08 \pm 60.97$ & $42.08 \pm 75.50$ & $24.33 \pm 22.57$ & 0.042 \\
\hline AUC $_{2 \mathrm{hGlucose}}$ & $1,022 \pm 184$ & $989 \pm 176$ & $1,072 \pm 185$ & 0.001 \\
\hline HbAlc $(\%)$ & $5.25 \pm 1.20$ & $5.33 \pm 1.25$ & $5.11 \pm 1.11$ & 0.192 \\
\hline Visceral adiposity index (VAI) & $2.14 \pm 1.00$ & $2.20 \pm 1.02$ & $2.01 \pm 0.97$ & 0.185 \\
\hline
\end{tabular}

Data are shown as Mean \pm SD or exact number and percent reported in parenthesis

treated with diet alone $(60 \%)$ or metformin (40\%); diabetic patients were treated with metformin alone $(58 \%)$ or in combination with other hypoglycemic drugs (sulphonylureas or glinides) $(42 \%)$.

The dose of metformin remained unchanged in all patients throughout the follow-up of the study, because no hypoglycemia or severe hyperglycemia were reported by the patients. Conversely, among diabetic patients who practiced sulphonylureas or glinides therapy, 50\% dose reduction was observed in 5 patients, because of slight hypoglycemic events.

Before metabolic evaluation, oral hypoglycemic drugs were suspended for 3-5 days and patients fasted for $12 \mathrm{~h}$, to avoid their effect on insulin sensitivity and secretion indexes [32].

Hormone and biochemical assays

During the study period GH levels were assayed by immunoradiometric and immunoenzymatic assays according with different availability. The sensitivity of the assays ranged $0.05-0.02 \mu \mathrm{g} / \mathrm{l}$. Serum IGF-1 was measured using immunoradiometric assays (Diagnostic System Laboratories Inc., Webster, TX). The normal ranges (for age) were: $180-625$ and $151-530(\leq 20), \quad 118-475$ and $118-450$ (21-30), 102-400 and 100-390 (31-40), 100-306 and 
96-228 (41-50), 95-270 and 90-250 (51-60), 88-250 and 82-200 (61-70), 78-200 and 68-188 $\mu \mathrm{g} / \mathrm{l}(\geq 70)$ for men and women respectively. The sensitivity of the assay was $0.8 \mu \mathrm{g} / \mathrm{l}$. The intra and interassay CVs were $3.4,3.0$ and $1.5 \%$, and $8.2,1.5$ and $3.7 \%$ for low, medium and high points on the standard curve, respectively. Serum insulin was measured by ELISA (DRG Instruments GmbH, Germany). The sensitivity of the method was $1 \mathrm{IU} / \mathrm{ml}$. The normal insulin range (IU/ml) was 5-19.

\section{Statistical analysis}

The Statistical Packages for Social Sciences SPSS version 17 was used for data analysis. Baseline and after-treatment characteristics were presented as Mean \pm standard deviation (SD) for continuous variables; rates and proportions were calculated for categorical data. All quantitative variables showed normal distribution (normality of distribution was assessed by means of the Kolmogorov-Smirnov test). Differences between groups in univariate analysis were detected by the unpaired Student's $t$ test for continuous variables and by the $\chi^{2}$-test and Fisher's exact test (when appropriate) for categorical variables. The differences between paired continuous variables (before and 1 year after therapy) were analyzed using the paired Student's $t$ test for continuous variables and the McNemar test for categorical variables.

Multiple linear regression analysis was performed to identify independent predictors of the continuous dependent variables ISI Matsuda and VAI score. Variables associated with the dependent variables on univariate analysis (Pearson's correlation; probability threshold, $P \leq 0.10)$ were included in three multivariate regression models. The categorical variable "Treatment" was coded to binary (dummy) variables (Surgery $=0 ; S A=1$ ) for inclusion in these models. A $P$ value of $<0.05$ was considered statistically significant.

\section{Results}

Baseline

Gender, age, duration of disease and tumor volume were similar in both groups.

Mean GH levels on diagnosis were $37.89 \pm 32.64$ and $37.47 \pm 29.98 \mu \mathrm{g} / \mathrm{l}(P=0.925)$, respectively in patients undergoing SRIF-RL or surgery, and no significant difference was found either for nadir GH after glucose load and IGF-1 levels.

Using the "National Cholesterol Education Program (NCEP-Adult Treatment Panel III, ATP III)" criteria [33], on diagnosis 85 acromegalic patients $(36.8 \%)$ were diagnosed as affected by MS, respectively, $27(11.7 \%)$ men and $58(25.1 \%)$ women, with higher prevalence in the age group over 50 years, especially in women $(39.6 \%$ vs. $72.7 \%$ ) (data not shown). No difference in MS prevalence was found grouping the patients on the basis of the treatment.

At baseline, 146 out of 231 patients (63.2\%) were classified as having normal glucose tolerance (NGT), 16 (6.9\%) IFG, 30 (13\%) IGT, 8 (3.5\%) combined IFG + IGT and $31(13.4 \%)$ overt DM. No difference in HbA1c levels was found between the two groups $(P=0.192)$.

The baseline clinical and biochemical features of the patients grouped according to the treatment assigned are shown in Table 1.

At 12 months of treatment

\section{Hormonal parameters}

After 12 months of treatment, as a whole, 102/231 patients $(44.1 \%)$ were classified as uncontrolled and 129/231 $(55.9 \%)$ as controlled [34]. In SRIF-RL and surgically treated patients, acromegaly was controlled in $89 / 151$ $(58.9 \%)$ and in $40 / 80$ (50\%) patients, respectively $(P=0.193)$.

Both therapies were effective in achieving control of the disease, leading to a similar reduction in mean fasting and nadir GH, AUC ${ }_{\mathrm{GH}}$ and IGF-1 levels (Table 2).

No difference in therapeutic efficacy was found between SRIF-RL and surgical treatment when we analyzed the delta of reduction of $\mathrm{GH} \quad(-34.1 \pm 31.4$ vs. $-33.6 \pm 30.8 \mu \mathrm{g} / \mathrm{l} ; P=0.904), \mathrm{AUC}_{\mathrm{GH}}(-3,356 \pm 3,236$ vs. $-3,036 \pm 2,761 ; P=0.468), \mathrm{GH}$ nadir $(-23.8 \pm 25.0$ vs. $-19.4 \pm 19.6 \mu \mathrm{g} / \mathrm{l} ; P=0.185)$ and IGF-1 ULN values $(-1.27 \pm 0.71$ vs. $-1.34 \pm 0.81 ; P=0.533)$.

\section{Metabolic syndrome (MS)}

After 12 months of therapy we found a significant decrease in prevalence of MS in SRIF-RL-treated patients, both in controlled $(P<0.001)$ and uncontrolled patients $(P=0.003)$. However, while in controlled SRIF-RL-treated patients the prevalence of each component of MS was reduced after 12 months, in uncontrolled ones WC and diastolic blood pressure remained unchanged. Conversely, in surgery patients, no reduction in the prevalence of MS was found, independently of achievement of control of the disease. Only a reduction in lipid parameters (triglycerides and HDL-cholesterol) was reported in all surgery patients, without any effect on blood pressure levels (Tables 2, 3). 
Table 2 Hormonal and metabolic parameters before and after treatment according to treatment: somatostatin analogues (somatotropin releaseinhibiting factor receptor ligand, SRIF-RL) and surgery

\begin{tabular}{|c|c|c|c|c|c|c|}
\hline & \multicolumn{3}{|c|}{$\begin{array}{l}\text { Acromegalics SRIF-RL-treated } \\
\text { (Group A: No 151) }\end{array}$} & \multicolumn{3}{|c|}{$\begin{array}{l}\text { Acromegalics surgery-treated } \\
\text { (Group B: No 80) }\end{array}$} \\
\hline & Basal & 12 months & & Basal & 12 months & \\
\hline Fasting GH $(\mu \mathrm{g} / \mathrm{l})$ & $37.9 \pm 32.6$ & $3.8 \pm 4.8$ & $<0.001$ & $37.5 \pm 30.0$ & $3.9 \pm 6.7$ & $<0.001$ \\
\hline $\mathrm{AUC}_{\mathrm{GH}}$ & $3,750 \pm 3,353$ & $393 \pm 509$ & $<0.001$ & $3,429 \pm 2,684$ & $393 \pm 777$ & $<0.001$ \\
\hline GH nadir $(\mu \mathrm{g} / \mathrm{l})$ & $26.8 \pm 25.7$ & $2.9 \pm 3.9$ & $<0.001$ & $22.2 \pm 19.4$ & $2.8 \pm 5.9$ & $<0.001$ \\
\hline IGF-1 ULN & $2.32 \pm 0.78$ & $1.04 \pm 0.48$ & $<0.001$ & $2.52 \pm 0.74$ & $1.18 \pm 0.48$ & $<0.001$ \\
\hline Normal tolerance & $98(64.9)$ & $103(68.2)$ & 0.596 & $47(58.8)$ & $57(71.3)$ & 0.089 \\
\hline Impaired fasting glucose (IFG) & $13(8.6)$ & $14(9.3)$ & 1 & $4(5)$ & $5(6.3)$ & 1 \\
\hline Impaired glucose tolerance (IGT) & $13(8.6)$ & $16(10.6)$ & 0.648 & $17(21.3)$ & $12(15)$ & 0.267 \\
\hline IFG + IGT & $4(2.6)$ & $13(8.6)$ & 0.049 & $4(5)$ & $4(5)$ & 1 \\
\hline Diabetes mellitus & $23(15.2)$ & $5(3.3)$ & $<0.001$ & $8(10)$ & $2(2,4)$ & 0.109 \\
\hline Metabolic syndrome & $59(39.1)$ & $26(17.2)$ & $<0.001$ & $26(32.5)$ & $21(26.3)$ & 0.383 \\
\hline Increased waist circumference & $29(19.2)$ & $20(13.2)$ & 0.012 & $24(30)$ & $33(41.3)$ & 0.004 \\
\hline Hypertriglyceridemia & $69(45.7)$ & $22(14.6)$ & $<0.001$ & $18(22.5)$ & $8(10)$ & 0.002 \\
\hline Low HDL cholesterol & $72(47.7)$ & $41(27.2)$ & $<0.001$ & $37(46.3)$ & $28(35)$ & 0.035 \\
\hline Increased systolic blood pressure or specific treatment & $106(70.2)$ & $66(43.7)$ & $<0.001$ & $58(72.5)$ & $58(72.5)$ & 1 \\
\hline $\begin{array}{l}\text { Increased diastolic blood pressure or specific } \\
\text { treatment }\end{array}$ & $73(48.3)$ & $52(34.4)$ & $<0.001$ & $42(52.5)$ & $38(47.5)$ & 0.454 \\
\hline Fasting glucose $(\mathrm{mmol} / \mathrm{l})$ & $5.63 \pm 0.96$ & $5.44 \pm 0.69$ & 0.018 & $5.26 \pm 0.58$ & $5.27 \pm 0.61$ & 0.991 \\
\hline $\mathrm{HbA1c}(\%)$ & $5.33 \pm 1.25$ & $5.02 \pm 0.67$ & 0.008 & $5.11 \pm 1.11$ & $5.36 \pm 0.61$ & 0.231 \\
\hline Homa IR & $4.16 \pm 2.70$ & $1.83 \pm 0.79$ & $<0.001$ & $4.46 \pm 2.41$ & $2.40 \pm 1.80$ & $<0.001$ \\
\hline Matsuda index & $3.27 \pm 1.67$ & $6.10 \pm 2.18$ & $<0.001$ & $2.67 \pm 0.84$ & $5.23 \pm 2.01$ & $<0.001$ \\
\hline $\mathrm{AUC}_{2 \mathrm{hIRI}}\left(\mathrm{mU}^{-1} 120 \mathrm{~min}\right)$ & $8,342 \pm 3,550$ & $5,117 \pm 2,614$ & $<0.001$ & $9,359 \pm 3,212$ & $5,604 \pm 2,621$ & $<0.001$ \\
\hline $\operatorname{AUC}_{2 \mathrm{hGLUCOSE}}\left(\mathrm{mmol} \mathrm{l}^{-1} 120 \mathrm{~min}\right)$ & $989 \pm 176$ & $923 \pm 168$ & $<0.001$ & $1,072 \pm 185$ & $967 \pm 175$ & $<0.001$ \\
\hline Insulinogenic index & $42.1 \pm 75.5$ & $26.6 \pm 42.1$ & 0.034 & $24.3 \pm 22.6$ & $18.4 \pm 22.6$ & 0.083 \\
\hline Visceral adiposity index (VAI) & $2.20 \pm 1.02$ & $1.80 \pm 0.81$ & $<0.001$ & $2.02 \pm 0.97$ & $1.81 \pm 0.71$ & 0.003 \\
\hline
\end{tabular}

Data are shown as Mean \pm SD or exact number and percent reported in parenthesis

\section{Glucose tolerance}

Table 4 shows the global cross of categories of glucose tolerance after 12 months of treatment. None of the patients with NGT at diagnosis developed diabetes after 12 months of treatment, regardless of achievement of disease control. Instead, a slight non-significant increase in NGT was detected both in SRIF-RL $(P=0.596$. $)$ and surgery-treated $(P=0.089)$ patients. A significant reduction in DM cases was observed during SRIF-RL treatment both in controlled $(P=0.003)$ and uncontrolled patients $(P=0.016)$, but with a concomitant increase in the combined IFG + IGT category in all SRIF-RL-treated $(P=0.049)$. By contrast, the prevalence of IGT, IFG and DM categories did not change after surgical treatment, regardless of achievement of disease control (Tables 2, 3).

\section{Insulin sensitivity and secretion indexes}

After 12 months, fasting glucose levels were reduced in the SRIF-RL-treated group $(P=0.018)$, whereas they did not change in the surgery-group. Grouping the patients according to disease control, SRIF-RL treatment improved fasting glucose only in controlled patients $(P<0.001)$. Conversely, a significant reduction in total glucose levels during OGTT (AUC $\mathrm{GLU}_{\mathrm{G}}$ ) was observed in both treatment groups $(P<0.001)$, but only acromegalic patients with controlled disease at 12 months showed a significant decrease in $\mathrm{AUC}_{\mathrm{GLU}}$, both after medical $(P<0.001)$ and surgical therapy $(P=0.001)$. HbA1c levels significantly decreased in SRIF-RL-treated $(P=0.008)$, while no significant variation was found in surgery patients $(P=0.231)$.

We observed a significant improvement in insulin resistance in both treatment groups, as shown by the decrease in Homa-IR and the increase in ISI Matsuda concomitance with a significant reduction in total insulin secretion $\left(\mathrm{AUC}_{\mathrm{INS}}\right)$, both in patients with controlled and uncontrolled acromegaly (all $P<0.001$ ) (Table 3). When we analyzed the variation in these parameters during therapy evaluating Delta ISI Matsuda and Delta $\mathrm{AUC}_{\mathrm{INS}}$, no difference was found between the two groups (data not shown). 
Table 3 Effects of different treatments: somatostatin analogues (somatotropin release-inhibiting factor receptor ligand, SRIF-RL) and surgery on metabolic parameters in uncontrolled and controlled patients

\begin{tabular}{|c|c|c|c|c|c|c|}
\hline & \multicolumn{6}{|c|}{ Uncontrolled Acromegalics (No. 102) } \\
\hline & \multicolumn{3}{|c|}{ Acromegalics SRIF-RL-treated (No. 62) } & \multicolumn{3}{|c|}{ Acromegalics surgery-treated (No. 40) } \\
\hline & Basal & 12 months & & Basal & 12 months & \\
\hline Normal tolerance (NGT) & $46(74.2)$ & $41(66.1)$ & 0.458 & $24(60)$ & $25(62.5)$ & 1 \\
\hline Impaired fasting glucose (IFG) & $4(6.5)$ & $7(11.3)$ & 0.549 & $3(7.5)$ & $2(5)$ & 1 \\
\hline Impaired glucose tolerance (IGT) & $5(8.1)$ & $9(14.5)$ & 0.289 & $8(20)$ & $8(20)$ & 1 \\
\hline $\mathrm{IFG}+\mathrm{IGT}$ & - & $5(8.1)$ & 0.063 & - & $3(7.5)$ & 0.250 \\
\hline Diabetes mellitus & $7(11.3)$ & - & 0.016 & $5(12.5)$ & $2(5)$ & 0.453 \\
\hline Metabolic syndrome & $25(40.3)$ & $11(17.7)$ & 0.003 & $14(35)$ & $14(35)$ & 1 \\
\hline Increased waist circumference & $8(12.9)$ & $6(9.7)$ & 0.625 & $12(30)$ & $16(40)$ & 0.125 \\
\hline Hypertriglyceridemia & $33(53.2)$ & $11(17.7)$ & $<0.001$ & $10(25)$ & $3(7.5)$ & 0.016 \\
\hline Low HDL cholesterol & $30(48.4)$ & 19 (30.6) & 0.003 & $23(57.5)$ & $18(45)$ & 0.180 \\
\hline $\begin{array}{l}\text { Increased systolic blood pressure } \\
\text { or specific treatment }\end{array}$ & $48(77.4)$ & 37 (59.7) & 0.027 & $30(75)$ & $34(85)$ & 0.219 \\
\hline $\begin{array}{l}\text { Increased diastolic blood pressure } \\
\text { or specific treatment }\end{array}$ & $39(62.9)$ & $35(56.5)$ & 0.454 & $22(55)$ & $23(57.5)$ & 1 \\
\hline Fasting glucose $(\mathrm{mmol} / \mathrm{l})$ & $5.31 \pm 0.87$ & $5.39 \pm 0.58$ & 0.593 & $5.27 \pm 0.56$ & $5.37 \pm 0.70$ & 0.434 \\
\hline HbAlc $(\%)$ & $5.29 \pm 1.28$ & $5.09 \pm 0.69$ & 0.253 & $5.11 \pm 0.84$ & $5.46 \pm 0.72$ & 0.085 \\
\hline Homa IR & $4.42 \pm 2.62$ & $1.79 \pm 0.55$ & $<0.001$ & $4.48 \pm 2.40$ & $2.63 \pm 1.63$ & $<0.001$ \\
\hline Matsuda index & $2.87 \pm 1.08$ & $5.59 \pm 1.89$ & $<0.001$ & $2.76 \pm 0.88$ & $4.73 \pm 1.83$ & $<0.001$ \\
\hline $\mathrm{AUC}_{2 \mathrm{hIRI}}\left(\mathrm{mU} \mathrm{l}^{-1} 120 \mathrm{~min}\right)$ & $8,863 \pm 3,050$ & $5,757 \pm 3,106$ & $<0.001$ & $8,882 \pm 2,961$ & $5,996 \pm 2,864$ & $<0.001$ \\
\hline $\operatorname{AUC}_{2 \mathrm{hGLUCOSE}}\left(\mathrm{mmol} \mathrm{l}^{-1} 120 \mathrm{~min}\right)$ & $986 \pm 116$ & $979 \pm 178$ & 0.729 & $1,056 \pm 191$ & $998.5 \pm 184$ & 0.090 \\
\hline Insulinogenic index & $22.82 \pm 11.58$ & $26.75 \pm 57.66$ & 0.631 & $26.24 \pm 29.12$ & $20.32 \pm 28.60$ & 0.322 \\
\hline $\mathrm{AUC}_{\mathrm{IRI}} / \mathrm{AUC}_{\mathrm{GLUCOSE}}$ & $9.06 \pm 3.18$ & $6.07 \pm 3.51$ & $<0.001$ & $8.79 \pm 3.55$ & $6.30 \pm 3.69$ & $<0.001$ \\
\hline \multirow[t]{4}{*}{ Visceral adiposity index (VAI) } & $2.21 \pm 0.79$ & $1.83 \pm 0.53$ & $<0.001$ & $2.08 \pm 1.01$ & $1.90 \pm 0.67$ & 0.126 \\
\hline & \multicolumn{6}{|c|}{ Controlled Acromegalics (No. 129) } \\
\hline & \multicolumn{3}{|c|}{ Acromegalics SRIF-RL-treated (No 89) } & \multicolumn{3}{|c|}{ Acromegalics surgery-treated (No 40) } \\
\hline & Basal & 12 months & & Basal & 12 months & \\
\hline Normal tolerance (NGT) & $52(58.4)$ & $62(69.7)$ & 0.089 & $23(57.5)$ & $32(80)$ & 0.035 \\
\hline Impaired fasting glucose (IFG) & $9(10.1)$ & $7(7.9)$ & 0.754 & $1(2.5)$ & $3(7.5)$ & 0.625 \\
\hline Impaired glucose tolerance (IGT) & $8(9)$ & $7(7.9)$ & 1 & $9(22.5)$ & $4(10)$ & 0.180 \\
\hline $\mathrm{IFG}+\mathrm{IGT}$ & $4(4.5)$ & $8(9)$ & 0.388 & $4(10)$ & $1(2.5)$ & 0.250 \\
\hline Diabetes mellitus & $16(18)$ & $5(5.6)$ & 0.003 & $3(7.5)$ & 0 & 0.250 \\
\hline Metabolic syndrome & $34(38.2)$ & $15(16.9)$ & $<0.001$ & $12(30)$ & $7(17.5)$ & 0.125 \\
\hline Increased waist circumference & $21(23.6)$ & $14(15.7)$ & 0.016 & $12(30)$ & $17(42.5)$ & 0.063 \\
\hline Hypertriglyceridemia & $36(40.4)$ & $11(12.4)$ & $<0.001$ & $8(20)$ & $5(12.5)$ & 0.250 \\
\hline Low HDL cholesterol & $42(47.2)$ & $22(24.7)$ & $<0.001$ & $14(35)$ & $10(25)$ & 0.219 \\
\hline $\begin{array}{l}\text { Increased systolic blood pressure } \\
\text { or specific treatment }\end{array}$ & $58(65.2)$ & $29(32.6)$ & $<0.001$ & $28(70)$ & $24(60)$ & 0.344 \\
\hline $\begin{array}{l}\text { Increased diastolic blood pressure } \\
\text { or specific treatment }\end{array}$ & $34(38.2)$ & $17(19.1)$ & $<0.001$ & $20(50)$ & $15(37.5)$ & 0.227 \\
\hline Fasting glucose $(\mathrm{mmol} / \mathrm{l})$ & $5.84 \pm 0.96$ & $5.47 \pm 0.75$ & $<0.001$ & $5.25 \pm 0.61$ & $5.16 \pm 0.50$ & 0.455 \\
\hline HbA1c $(\%)$ & $5.35 \pm 1.23$ & $4.97 \pm 0.65$ & 0.014 & $5.23 \pm 1.38$ & $5.27 \pm 0.47$ & 0.867 \\
\hline Homa IR & $4 \pm 2.74$ & $1.86 \pm 0.91$ & $<0.001$ & $4.43 \pm 2.45$ & $2.18 \pm 1.95$ & $<0.001$ \\
\hline Matsuda index & $3.52 \pm 1.91$ & $6.41 \pm 2.30$ & $<0.001$ & $2.59 \pm 0.80$ & $5.74 \pm 2.07$ & $<0.001$ \\
\hline $\mathrm{AUC}_{2 \mathrm{hIRI}}\left(\mathrm{mU} \mathrm{l}^{-1} 120 \mathrm{~min}\right)$ & $8,025 \pm 3,808$ & $4,727 \pm 2,194$ & $<0.001$ & $9,847 \pm 3,419$ & $5,202 \pm 2,315$ & $<0.001$ \\
\hline $\operatorname{AUC}_{2 \mathrm{hGLUCOSE}}\left(\mathrm{mmol} \mathrm{l}^{-1} 120 \mathrm{~min}\right)$ & $990 \pm 205$ & $888 \pm 153$ & $<0.001$ & $1,088 \pm 179$ & $936 \pm 162$ & 0.001 \\
\hline
\end{tabular}


Table 3 continued

\begin{tabular}{|c|c|c|c|c|c|c|}
\hline & \multicolumn{6}{|c|}{ Controlled Acromegalics (No. 129) } \\
\hline & \multicolumn{3}{|c|}{ Acromegalics SRIF-RL-treated (No 89) } & \multicolumn{3}{|c|}{ Acromegalics surgery-treated (No 40) } \\
\hline & Basal & 12 months & & Basal & 12 months & \\
\hline Insulinogenic index & $53.99 \pm 93.88$ & $26.52 \pm 28.99$ & 0.010 & $22.42 \pm 13.28$ & $16.54 \pm 14.61$ & 0.082 \\
\hline $\mathrm{AUC}_{\mathrm{IRI}} / \mathrm{AUC}_{\mathrm{GLUCOSE}}$ & $8.51 \pm 5.39$ & $5.43 \pm 2.61$ & $<0.001$ & $9.37 \pm 3.70$ & $5.66 \pm 2.63$ & $<0.001$ \\
\hline Visceral adiposity index (VAI) & $2.20 \pm 1.16$ & $1.78 \pm 0.96$ & $<0.001$ & $1.95 \pm 0.93$ & $1.71 \pm 0.75$ & 0.004 \\
\hline
\end{tabular}

Data are shown as Mean $\pm \mathrm{SD}$ or exact number and percent reported in parenthesis

In addition, grouping the whole cohort of patients only according to disease control status, we found a greater increase in ISI Matsuda $(6.19 \pm 2.22$ vs. $5.20 \pm 1.90$; $P=0.001)$ and a greater decrease in $\mathrm{AUC}_{\mathrm{INS}}(4,872 \pm$ $2,215$ vs. $5,867 \pm 2,983 ; P=0.007)$ in controlled than uncontrolled patients (data not shown). Only in the controlled SRIF-RL-treated there was also a significant reduction in HbAlc levels $(P=0.014)$ and early insulin secretion (insulinogenic index) $(P=0.010)$, while no difference was found in surgery patients (Tables 2, 3; Fig. 2).

Table 4 shows changes in glucose tolerance during the study.

\section{Adipose tissue dysfunction}

A significant decrease in VAI score was observed in the SRIF-RL-treated group, in patients with both controlled $(P<0.001)$ and uncontrolled disease $(P<0.001)$. In surgery patients a reduction in VAI score was observed only when disease control was achieved $(P=0.004)$ (Tables 2 , 3; Fig. 2).

Table 4 Contingency table showing the global cross of categories of glucose tolerance after 12 months of treatment

\section{Multivariate analysis}

To detect which variable independently influences insulin sensitivity (ISI Matsuda), and VAI after 12 months of therapy, we constructed two different models of multiple linear regression for acromegalic patients grouped according to the different treatments (Table 5; Fig. 3).

ISI Matsuda showed a significant independent correlation with IGF-1 ULN values $(\beta=-0.258 ; P=0.001)$ and VAI score $(\beta=-0.430 ; P<0.001)$. No correlation was detected between ISI Matsuda and therapy.

The VAI score was independently correlated with age $(\beta=0.247 ; P<0.001)$ and IGF-1 ULN values $(\beta=0.183$; $P=0.004)$.

\section{Discussion}

This retrospective study compared the metabolic effects of 12 months of first-line SRIF-RL or surgical therapy in a large cohort of acromegalic patients in relation to the achievement of disease control.

\begin{tabular}{|c|c|c|c|c|c|c|}
\hline & \multicolumn{6}{|c|}{12 months } \\
\hline & NGT & IFG & IGT & $\mathrm{IFG}+\mathrm{IGT}$ & DM & Total \\
\hline \multicolumn{7}{|c|}{ Patients treated with somatostatin analogues } \\
\hline \multicolumn{7}{|l|}{ Basal } \\
\hline NGT & 73 & 10 & 8 & 5 & 2 & 98 \\
\hline IFG & 9 & 3 & 1 & 0 & 0 & 13 \\
\hline IGT & 5 & 1 & 6 & 1 & 0 & 13 \\
\hline $\mathrm{IFG}+\mathrm{IGT}$ & 3 & 0 & 1 & 0 & 0 & 4 \\
\hline DM & 13 & 0 & 0 & 7 & 3 & 23 \\
\hline Total & 103 & 14 & 16 & 13 & 5 & 151 \\
\hline \multicolumn{7}{|c|}{ Patients treated with surgery } \\
\hline \multicolumn{7}{|l|}{ Basal } \\
\hline NGT & 37 & 4 & 5 & 1 & 0 & 47 \\
\hline IFG & 3 & 0 & 0 & 0 & 1 & 4 \\
\hline IGT & 9 & 0 & 7 & 1 & 0 & 17 \\
\hline $\mathrm{IFG}+\mathrm{IGT}$ & 3 & 0 & 0 & 1 & 0 & 4 \\
\hline DM & 5 & 1 & 0 & 1 & 1 & 8 \\
\hline Total & 57 & 5 & 12 & 4 & 2 & 80 \\
\hline
\end{tabular}


Fig. 2 Variation (on the left) and Delta (on the right) of changed insulin sensitivity (ISI Matsuda), insulin secretion $\left(\mathrm{AUC}_{\mathrm{IRI}}\right)$ and visceral adipose function (VAI) before and after 12 months of somatostatin analogues (somatotropin release-inhibiting factor receptor ligand, SRIF-RL) and surgical treatment
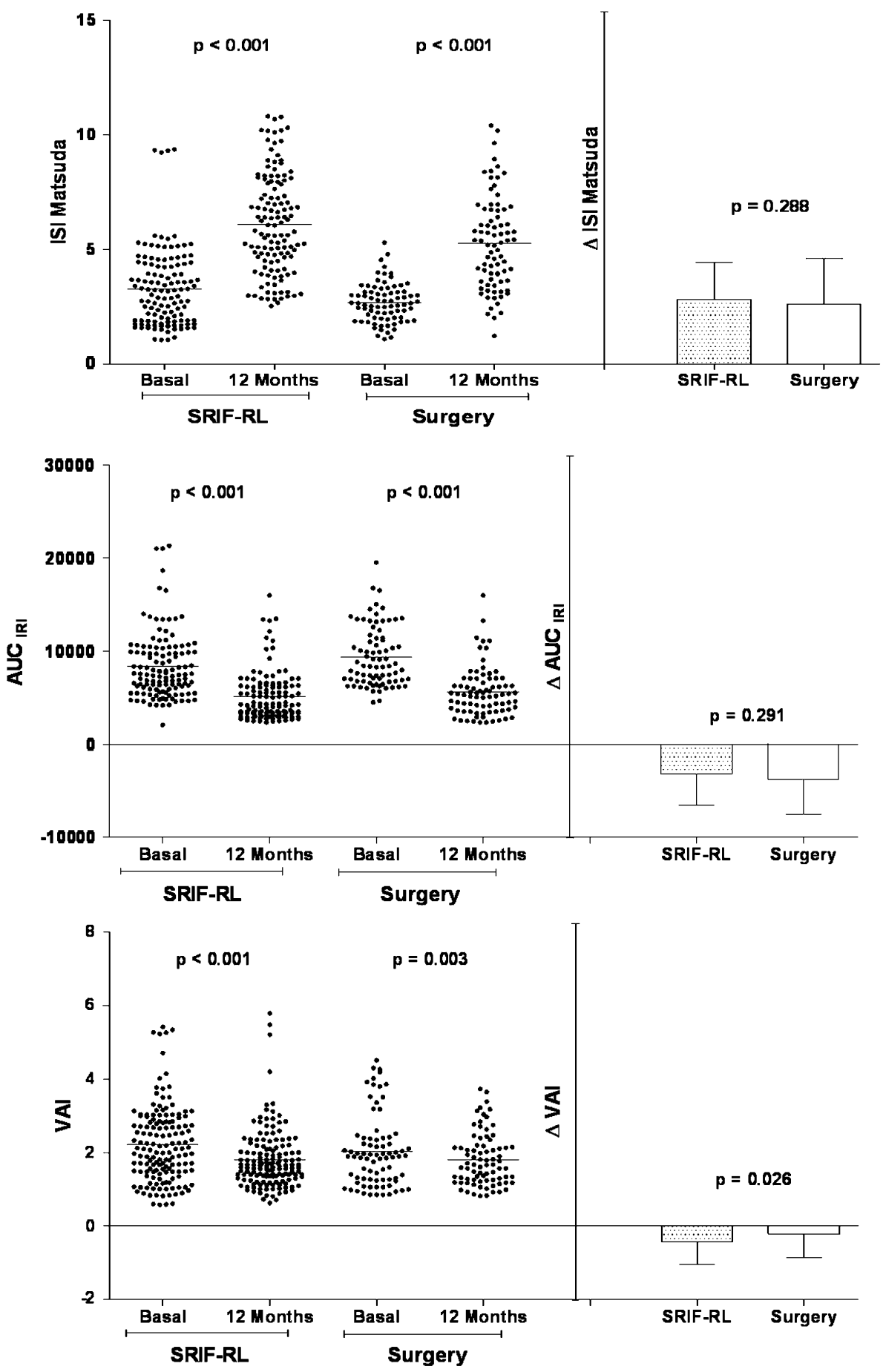

Although the efficacy in the reduction in GH and IGF-1 levels proved similar, the results of this study showed different metabolic effects in the two groups. MS has been described in acromegaly, but studies analyzing the prevalence of possible MS clusters in accordance with the ATP III criteria are still not fully available. In our study SRIF-RL proved to be more efficacious than surgery in improving all MS parameters and reducing fasting glucose, $\mathrm{HbA} 1 \mathrm{c}$ and the prevalence of DM when disease control was achieved. We describe the same effects on fasting glucose and HbAlc as well as in another comparative study analyzing a smaller cohort of patients grouped in relation to the different treatments $[22,23]$. 


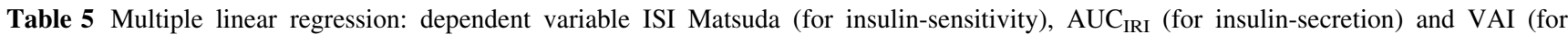
visceral adipose function) after 12 months of treatment

\begin{tabular}{|c|c|c|c|c|c|}
\hline \multirow[t]{2}{*}{ Independent variables at 12 months } & \multicolumn{2}{|c|}{ Univariate analysis } & \multicolumn{3}{|c|}{ Multivariate analysis } \\
\hline & $r$ & $P$ & $\beta$ & SE & $P$ \\
\hline \multicolumn{6}{|c|}{ Dependent variable: ISI Matsuda at 12 months } \\
\hline Age & -0.140 & 0.045 & -0.077 & 0.010 & 0.299 \\
\hline Duration of disease & -0.110 & 0.116 & - & - & - \\
\hline BMI & -0.100 & 0.153 & - & - & - \\
\hline WC & -0.143 & 0.041 & 0.044 & 0.016 & 0.534 \\
\hline Nadir GH & -0.162 & 0.020 & 0.925 & 0.273 & 0.125 \\
\hline AUC GH & -0.174 & 0.013 & -0.947 & 0.002 & 0.117 \\
\hline IGF1 (ULN) & -0.372 & $<0.001$ & -0.258 & 0.236 & 0.001 \\
\hline VAI & -0.488 & $<0.001$ & -0.430 & 0.172 & $<0.001$ \\
\hline Treatment* & 0.188 & 0.007 & 0.122 & 0.275 & 0.051 \\
\hline \multicolumn{6}{|c|}{ Dependent variable: $\mathrm{AUC}_{\mathrm{IRI}}$ at 12 months } \\
\hline Age & 0.031 & 0.622 & - & - & - \\
\hline Duration of disease & 0.023 & 0.740 & - & - & - \\
\hline BMI & -0.035 & 0.619 & - & - & - \\
\hline WC & -0.040 & 0.568 & - & - & - \\
\hline Nadir GH & 0.029 & 0.675 & - & - & - \\
\hline AUC GH & 0.025 & 0.722 & - & - & - \\
\hline IGF1 (ULN) & 0.194 & 0.005 & 0.154 & 353 & 0.025 \\
\hline VAI & 0.254 & $<0.001$ & 0.227 & 221 & 0.001 \\
\hline Treatment* & -0.095 & 0.173 & - & - & - \\
\hline \multicolumn{6}{|l|}{ Dependent variable: VAI at 12 months } \\
\hline Age & 0.232 & $<0.001$ & 0.247 & 0.003 & $<0.001$ \\
\hline Duration of disease & 0.071 & 0.284 & - & - & - \\
\hline Nadir GH & 0.014 & 0.839 & - & - & - \\
\hline AUC GH & 0.011 & 0.877 & - & - & - \\
\hline IGF1 (ULN) & 0.163 & 0.013 & 0.183 & 0.101 & 0.004 \\
\hline Treatment* & -0.004 & 0.955 & - & - & - \\
\hline
\end{tabular}

* Categorical variable coded to binary (dummy) variables: Surgery $=0$; Somatostatin analogues $=1$

Unchanged blood pressure levels were found in the surgical group in comparison to SRIF-RL-treated patients, partially confirming previous findings which demonstrated greater benefit for cardiac performance in SRIF-RL-treated acromegalic patients [22]. As regards WC, we found a significant decrease after SRIF-RL and surprisingly an increase in surgically treated patients. Although our findings remain speculative, we can hypothesize a different compliance of patients in the two groups: probably the patients receiving medical therapy were followed up more closely, while surgery-treated patients were less compliant in follow-up.

The effects of acromegalic treatment on glucose and insulin homeostasis have been analyzed in several studies. Although a significant increase in insulin sensitivity is always observed after SRIF-RL treatment, glucose metabolism frequently proves to be unchanged [21] or deteriorated $[23,35,36]$, associated with a delayed insulin secretion peak during OGTT [14, 37]. Increased glucose levels in both controlled and uncontrolled patients were reported during SRIF-RL therapy and not after surgery [18] and surgical removal of pituitary tumors proved effective in improving glucose abnormalities [38]. These findings support the hypothesis that the reduction in insulin secretion after SRIF-RL could be a consequence of a suppressive effect on $\beta$-cell secretion rather than being an indirect consequence of improved insulin sensitivity. Conversely, an improvement in insulin sensitivity without worsening of glucose levels during octreotide therapy was demonstrated [39]. However, the primary outcome measures considered in the previous studies were fasting glucose and insulin levels or glucose tolerance status categories [40] and insulin homeostasis was assessed mainly by sensitivity (Homa-IR) and secretion (Homa- $\beta$ ) indexes based on fasting glucose and insulin levels. In the current study, for better evaluation of insulin sensitivity, we also considered 
Fig. 3 Independent variables influencing insulin sensitivity (ISI Matsuda), insulin secretion $\left(\mathrm{AUC}_{\mathrm{IRI}}\right)$ and visceral adipose function (VAI)
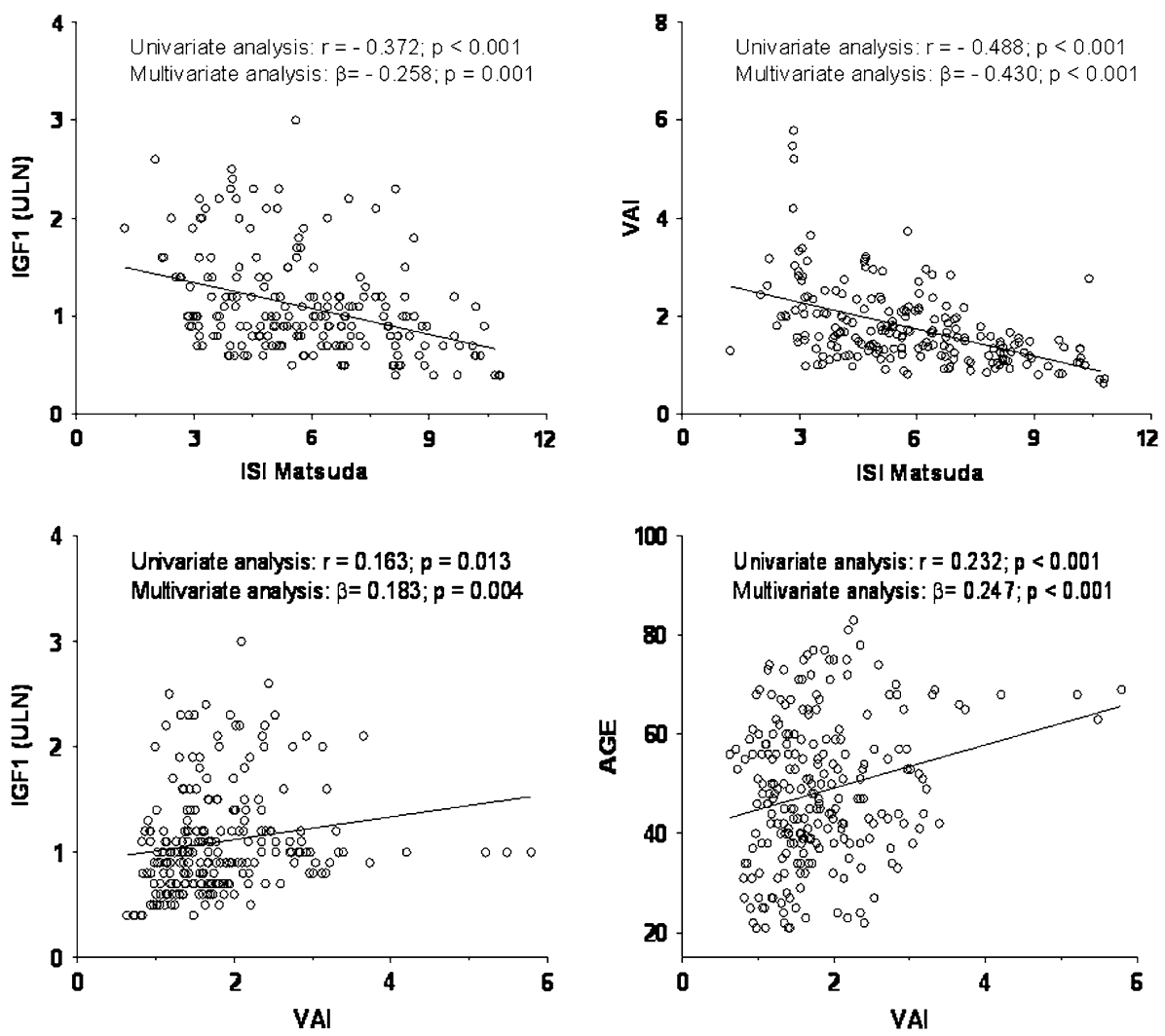

ISI Matsuda, a stimulated and more reliable index deriving from OGTT, recently suggested to be more closely correlated with insulin sensitivity than the other indexes deriving from fasting measurements and Homa-IR [41].

Moreover, to assess insulin secretion, we used two other parameters: an early (insulinogenic index) and a total $\left(\mathrm{AUC}_{\mathrm{INS}}\right)$ secretion index. Our data showed that acromegaly treatment, both medical and surgical, improves insulin sensitivity and reduces insulin levels, in the absence of any deterioration in glucose tolerance. Notably, we found a significant negative correlation between ISI Matsuda and IGF-1 ULN, showing a greater improvement in insulin-sensitivity parallel to decreased IGF-1 levels. These data are consistent with a recent meta-analysis on the clinical impact of SRIF-RL on glucose metabolism [40]. Therefore, the hormonal control of acromegaly proves to be the main determinant of insulin-sensitivity, regardless of the treatment practiced. In controlled SRIF-RL-treated patients we found that both early (insulinogenic index) and total $\left(\mathrm{AUC}_{\mathrm{INS}}\right)$ insulin secretion decreased after 12 months of therapy, while in surgical patients only total secretion was influenced. These data are partially in agreement with the study by Ronchi et al. [18], which showed a significant basal insulin secretion decrease in all SRIF-RL-treated patients regardless of disease control, but with a concomitant insulinogenic index reduction only in controlled SRIF-RL-treated patients. For this reason, the main role determined by disease control appears to be played by the reduction in compensatory hyperinsulinism, secondary to the improvement in insulin sensitivity. Furthermore, in controlled patients, SRIF-RL exerts an additional direct effect, as demonstrated by the more pronounced early insulin secretion reduction (pre-SRIF-RL insulinogenic index $54.0 \pm 93.9 ;$ post-SRIF-RL $26.5 \pm 29.0 ; \quad P=$ 0.010 ) compared to surgery (pre-surgery insulinogenic index $22.4 \pm 13.3$; post-surgery $16.5 \pm 14.6 ; P=0.082$ ) (Table 3 ). When we analyzed the change of insulin secretion and sensitivity indexes after 12 months of treatment in each glucose tolerance category, even if the patients did not cross category, we found a significant increase in insulin sensitivity (ISI Matsuda) in all groups and a decrease in total insulin secretion (AUC $\mathrm{INS}_{\mathrm{I}}$ ) in NGT, IFG and DM (data not shown).

In our study, in addition to evaluating metabolic effects based on insulin homeostasis, we also considered an innovative aspect based on visceral adiposity function indirectly expressed by VAI. Our data show that active acromegaly is strongly associated with visceral adiposity dysfunction and both therapies are able to improve it, as demonstrated by the significant VAI decrease after 12 months. Seeing that IGF-1, VAI and ISI Matsuda influence one another, VAI could represent an additional link between disease control and insulin sensitivity. In this connection, the most insulin-resistant patients were those 
with uncontrolled disease and higher VAI. GH and IGF-1 are well recognized as important regulators of metabolism and body composition with complex interaction, with predominant GH lipolytic and insulin-resistant effect when at supra-physiological levels, as in acromegaly [42, 43]. Adipose tissue lipolysis seems to occur mainly through stimulation of $\beta$-adrenergic receptors, the adenylate cyclase system and hormone-sensitive lipase expression [44, 45]. In this connection, in effect in acromegaly a lipotoxicity condition has been described [46], with increased intermuscular adipose tissue despite a reduction in visceral and subcutaneous adipose tissue. This evidence may be explained on the basis of the complex mechanisms of the lipotoxicity of lean tissues proposed by Unger et al. [47]. In acromegalic patients a pathophysiological condition similar to that in subjects with lipodystrophy, a condition characterized by severe insulin-resistance and lipotoxicity of lean tissues, could be hypothesized [48, 49] and VAI may indirectly express this condition. However, further studies on the interaction between the GH-IGF-1 axis and adipocytokines could clarify these mechanisms.

In conclusion, in the current study, which included one of the largest cohorts of acromegalic patients so far studied for glucose metabolism, we demonstrated that SRIF-RL and surgical treatment can be safely employed as first-line therapy for acromegaly, without any untoward effects on glucose tolerance and disease control is the main determinant of beneficial effects on general features of insulin sensitivity.

Acknowledgments This study has been supported in part by grants of Italian Minister of Research (MIUR)”, no. 2003068735.

Conflict of interest Annamaria Colao is recipient of unrestricted support from Ipsen, Novartis and Pfizer for studies in neuroendocrinology. The other authors have nothing to disclose.

Ethical standards The authors declare that the experiments comply with the current Italian laws.

\section{References}

1. Melmed S (2006) Medical progress: acromegaly. N Engl J Med 355:2558-2573

2. Melmed S (2009) Acromegaly pathogenesis and treatment. J Clin Invest 119(11):3189-3202

3. Colao A, Ferone D, Marzullo P, Lombardi G (2004) Systemic complications of acromegaly: epidemiology, pathogenesis and management. Endocr Rev 25:102-152

4. Giustina A, Casanueva FF, Cavagnini F, Chanson P, Clemmons D, Frohman LA, Gaillard R, Ho K, Jaquet P, Kleinberg DL, Lamberts SW, Lombardi G, Sheppard M, Strasburger CJ, Vance ML, Wass JA, Melmed S (2003) Diagnosis and treatment of acromegaly complications. J Endocrinol Invest 26:1242-1247

5. Jayasena C, Comninos A, Clarke H, Donaldson M, Meeran K, Dhillo W (2011) The effects of long-term GH and IGF-1 exposure on the development of cardiovascular, cerebrovascular and metabolic co-morbidities in treated patients with acromegaly. Clin Endocrinol (Oxf) 75:220-225

6. Colao A, Baldelli R, Marzullo P, Ferretti F, Ferone D, Gargiulo P, Petretta M, Tamburrano G, Lombardi G, Liuzzi A (2000) Systemic hypertension and impaired glucose tolerance are independently correlated to the severity of the acromegalic cardiomyopathy. J Clin Endocrinol Metab 85:193-199

7. Sherlock M, Woods C, Sheppard MC (2011) Medical therapy in acromegaly. Nat Rev Endocrinol 7(5):291-300

8. Melmed S, Casanueva FF, Cavagnini F, Chanson P, Frohman L, Grossman A, Ho K, Kleinberg D, Lamberts S, Laws E, Lombardi G, Vance ML, von Werder K, Wass J, Giustina A, for the Acromegaly Treatment Consensus Workshop Participants' (2002) Guidelines for acromegaly management. J Clin Endocrinol Metab 87:4054-4058

9. Colao A, Auriemma RS, Lombardi G, Pivonello R (2011) Resistance to somatostatin analogs in acromegaly. Endocr Rev 32:247-271

10. Mercado M, Borges F, Bouterfa H, Chang TC, Chervin A, Farrall AJ, Patocs A, Petersenn S, Podoba J, Safari M, Wardlaw J, SMS995B2401 Study Group (2007) A prospective, multicentre study to investigate the efficacy, safety and tolerability of octreotide LAR (long-acting repeatable octreotide) in the primary therapy of patients with acromegaly. Clin Endocrinol (Oxf) 66(6):859-868

11. Alberti KGMM, Christensen SE, Christensen NJ, Hansen AP, Iversen J, Lundbaek K, Seyer-Hansen K, Orskov H (1973) Inhibition of insulin secretion by somatostatin. Lancet 2:12991301

12. Johansson C, Wisen O, Efendic S, Uvnas-Wallenstein K (1981) Effect of somatostatin on gastrointestinal propagation and absorption of oral glucose in man. Digestion 22:126-137

13. Steffin B, Gutt B, Bidlingmaier M, Dieterle C, Oltmann F, Schopohl J (2006) Effects of the long-acting somatostatin analogue Lanreotide Autogel on glucose tolerance and insulin resistance in acromegaly. Eur J Endocrinol 155:73-78

14. Pereira AM, Biermasz NR, Roelfsema F, Romijn JA (2005) Pharmacologic therapies for acromegaly. A review of their effects on glucose metabolism and insulin resistance. Treat Endocrinol 4:43-53

15. Saitoh Y, Ohnishi T, Arita N (2000) Impaired beta-cell function in the presence of reduced insulin sensitivity determines glucose tolerance status in acromegalic patients. Clin Endocrinol (Oxf) 52:549-555

16. Karaca Z, Tanriverdi F, Elbuken G, Cakir I, Donmez H, Selcuklu A, Durak A, Dokmetas H, Colak R, Unluhizarci K, Kelestimur F (2011) Comparison of primary octreotide-LAR and surgical treatment in newly diagnosed patients with acromegaly. Clin Endocrinol (Oxf). doi:10.1111/j.1365-2265.2011.04106.x [Epub ahead of print]

17. Tzanela M, Vassiliadi DA, Gavalas N, Szabo A, Margelou E, Valatsou A, Vassilopoulos C (2011) Glucose homeostasis in patients with acromegaly treated with surgery or somatostatin analogues. Clin Endocrinol (Oxf). doi:10.1111/j.1365-2265. 2011.03996.x [Epub ahead of print]

18. Ronchi CL, Varca V, Beck-Peccoz P, Orsi E, Donadio F, Baccarelli A, Giavoli C, Ferrante E, Lania A, Spada A, Arosio M (2006) Comparison between six-year therapy with long-acting somatostatin analogs and successful surgery in acromegaly: effects on cardiovascular risk factors. J Clin Endocrinol Metab 91:121-128

19. Chen HS, Wu TE, Jap TS, Hsiao LC, Lin HD, Lee SH, Lin SH (2011) Effects of long-acting release octreotide on glucose homeostasis in acromegaly patients after trans-sphenoidal surgery. Horm Metab Res 43:433-439

20. Cambuli VM, Galdiero M, Mastinu M, Pigliaru F, Auriemma RS, Ciresi A, Pivonello R, Amato M, Giordano C, Mariotti S, Colao 
A, Baroni MG (2011) Glycometabolic control in acromegalic patients with diabetes: a study of the effects of different treatments for GH excess and for hyperglycaemia. J Endocrinol Invest [Epub ahead of print]

21. Colao A, Auriemma RS, Savastano S, Galdiero M, Grasso LF, Lombardi G, Pivonello R (2009) Glucose tolerance and somatostatin analog treatment in acromegaly: a 12-month study. J Clin Endocrinol Metab 94:2907-2914

22. Colao A, Pivonello R, Galderisi M, Cappabianca P, Auriemma RS, Galdiero M, Cavallo LM, Esposito F, Lombardi G (2008) Impact of treating acromegaly first with surgery or somatostatin analogs on cardiomyopathy. J Clin Endocrinol Metab 93: 2639-2646

23. Colao A, Auriemma RS, Galdiero M, Cappabianca P, Cavallo LM, Esposito F, Grasso LF, Lombardi G, Pivonello R (2009) Impact of somatostatin analogs versus surgery on glucose metabolism in acromegaly: results of a 5-years observational, open, prospective study. J Clin Endocrinol Metab 94:528-537

24. Amato MC, Giordano C, Galia M, Criscimanna A, Vitabile S, Midiri M, Galluzzo A, For the AlkaMeSy Study Group (2010) Visceral Adiposity Index (VAI): a reliable indicator of visceral fat function associated with cardiometabolic risk. Diabetes Care 33:920-922

25. Colao A, Martino E, Cappabianca P, Cozzi R, Scanarini M, Ghigo E, The participants of the A.L.I.C.E. (Acromegaly primary medical treatment Learning and Improvement with Continuous medical Education) Study Group (2006) J Endocrinol Invest 29:1017-1020

26. Colao A, Lombardi G (1998) Growth hormone and prolactin excess. Lancet 352:1455-1461

27. Giustina A, Chanson P, Bronstein MD, Klibanski A, Lamberts S, Casanueva FF, Trainer P, Ghigo E, Ho K, Melmed S, Acromegaly Consensus Group (2010) A consensus on criteria for cure of acromegaly. J Clin Endocrinol Metab 95:3141-3148

28. American Association of Clinical Endocrinologists (2000) The American Association of Clinical Endocrinologists Medical Guidelines for the Management of Diabetes Mellitus: the AACE system of intensive diabetes self-management-2000 update. Endocr Pract 6(1):43-84

29. Matthews DR, Hosker JP, Rudenski AS, Naylor BA, Treacher DF, Turner RC (1985) Homeostasis model assessment: insulin resistance and b-cell function from fasting plasma glucose and insulin in man. Diabetologia 28:412-419

30. Matsuda M, De Fronzo RA (2001) Insulin sensitivity indices obtained from oral glucose tolerance testing: comparison with the euglycemic insulin clamp. Diabetes Care 24:460-464

31. Stumvoll M, Mitrakou A, Pimenta W, Jenssen T, Yki-Jarvinen H, Van Haeften T, Renn W, Gerich J (2000) Use of the oral glucose tolerance test to assess insulin release and insulin sensitivity. Diabetes Care 23:295-301

32. Tucker GT, Casey C, Phillips PJ, Connor H, Ward JD, Woods HF (1981) Metformin kinetics in healthy subjects and in patients with diabetes mellitus. Br J Clin Pharmacol 12:235-246

33. Expert Panel on Detection, Evaluation, and Treatment of High Blood Cholesterol in Adults (Adult Treatment Panel III) (2001) Expert panel on detection, evaluation, and treatment of high blood cholesterol in adults: executive summary of the third report of the National Cholesterol Education Program (NCEP). J Am Med Assoc 285:2486-2497
34. Giustina A, Barkan A, Casanueva FF, Cavagnini F, Frohman L, Ho K, Veldhuis J, Wass J, Von Werder K, Melmed S (2000) Criteria for cure of acromegaly: a consensus statement. J Clin Endocrinol Metab 85:526-529

35. Colao A, Ferone D, Marzullo P, Cappabianca P, Cirillo S, Boerlin V, Lancrajan I, Lombardi G (2001) Long-term effects of depot long-acting somatostatin analog octreotide on hormone levels and tumor mass in acromegaly. J Clin Endocrinol Metab 86: 2779-2786

36. Arosio M, Macchelli S, Rossi CM, Casati G, Biella O, Foglia G (1995) Effects of treatment with octreotide in acromegalic patients-a multicenter Italian study. Italian Multicenter Octreotide Study Group. Eur J Endocrinol 133:430-439

37. Baldelli R, Battista C, Leonetti F, Ghiggi MR, Ribaudo MC, Paoloni A, D'Amico E, Ferretti E, Baratta R, Liuzzi A, Trischitta V, Tamburrano G (2003) Glucose homeostasis in acromegaly: effects of long-acting somatostatin analogues treatment. Clin Endocrinol 59:492-499

38. Sonksen PH, Greenwood FC, Ellis JP, Lowy C, Rutherford A, Nabarro JD (1967) Changes of carbohydrate tolerance in acromegaly with progress of the disease and in response to treatment. J Clin Endocrinol Metab 27:1418-1430

39. Ho KK, Jenkins AB, Furler SM, Borkman M, Chisholm DJ (1992) Impact of octreotide, a long-acting somatostatin analogue, on glucose tolerance and insulin sensitivity in acromegaly. Clin Endocrinol (Oxf) 36:271-279

40. Mazziotti G, Floriani I, Bonadonna S, Torri V, Chanson P, Giustina A (2009) Effects of somatostatin analogs on glucose homeostasis: a meta-analysis acromegaly studies. J Clin Endocrinol Metab 94:1500-1508

41. Lorenzo C, Haffner SM, Stancáková A, Laakso M (2010) Relation of direct and surrogate measures of insulin resistance to cardiovascular risk factors in nondiabetic Finnish offspring of type 2 diabetic individuals. J Clin Endocrinol Metab 95: 5082-5090

42. Bengtsson BA, Brummer RJ, Bosaeus I (1990) Growth hormone and body composition. Horm Res 33(Suppl 4):19-24

43. Clemmons DR (2002) Roles of insulin-like growth factor-I and growth hormone in mediating insulin resistance in acromegaly. Pituitary 5:181-183

44. Richelsen B (1997) Action of growth hormone in adipose tissue. Horm Res 48(Suppl 5):105-110

45. Yang S, Mulder H, Holm C, Eden S (2004) Effects of growth hormone on the function of beta-adrenoceptor subtypes in rat adipocytes. Obes Res 12:330-339

46. Freda PU, Shen W, Heymsfield SB, Reyes-Vidal CM, Geer EB, Bruce JN, Gallagher D (2008) Lower visceral and subcutaneous but higher intermuscular adipose tissue depots in patients with growth hormone and insulin-like growth factor I excess due to acromegaly. J Clin Endocrinol Metab 93:2334-2343

47. Unger RH, Scherer PE (2010) Gluttony, sloth and the metabolic syndrome: a roadmap to lipotoxicity. Trends Endocrinol Metab 21:345-352

48. Garg A (2000) Lipodystrophies. Am J Med 108:143-152

49. Asilmaz E, Cohen P, Miyazaki M, Dobrzyn P, Ueki K, Fayzikhodjaeva G, Soukas AA, Kahn CR, Ntambi JM, Socci ND, Friedman JM (2004) Site and mechanism of leptin action in a rodent form of congenital lipodystrophy. J Clin Invest 113: $414-424$ 